\title{
PULSED MODERATOR STUDIES USING A TIME FOCUSSED CRYSTAL SPECTROMETER
}

\author{
K. F. GRAHAM and J. M. CARPENTER
}

Department of Nuclear Engineering, The University of Michigan, Ann Arbor, Michigan 48105, U.S.A.

Received 16 April 1970

\begin{abstract}
A technique is reported for measuring the joint time and energy dependence of the rate of emission of slow neutrons emerging from moderators pulsed with fast neutrons. A crystal spectrometer selects neutrons of a given energy and the leakage flux of that energy is examined as a function of time. The spectrometer is arranged to be time-focussed; time resolution is decoupled from energy, spatial and angular resolution. Time-focussing
\end{abstract}

\section{Introduction}

We report a technique for measuring the joint time and energy dependence of the rate of emission of slow neutrons emerging from moderators pulsed with fast neutrons. A crystal spectrometer is used to select neutrons of a given energy and the leakage flux of that energy is examined as a function of time. The crystal spectrometer is arranged to be time-focussed; time resolution is decoupled from energy, spatial and angular resolution. The time-focussing fcature cnables measurements to be made using much less intense neutron sources than have been used by earlier workers. Adequate counting rates are obtained by sampling large source area, and large angle and energy widths, while good time resolution is maintained by time focussing.

Measurements of the moderator time-energy distributions are required for design of intense pulsed slow neutron sources, and are useful as demanding tests of scattering kernels and neutron transport calculational methods. Fig. 1 illustrates the basic quantity to be
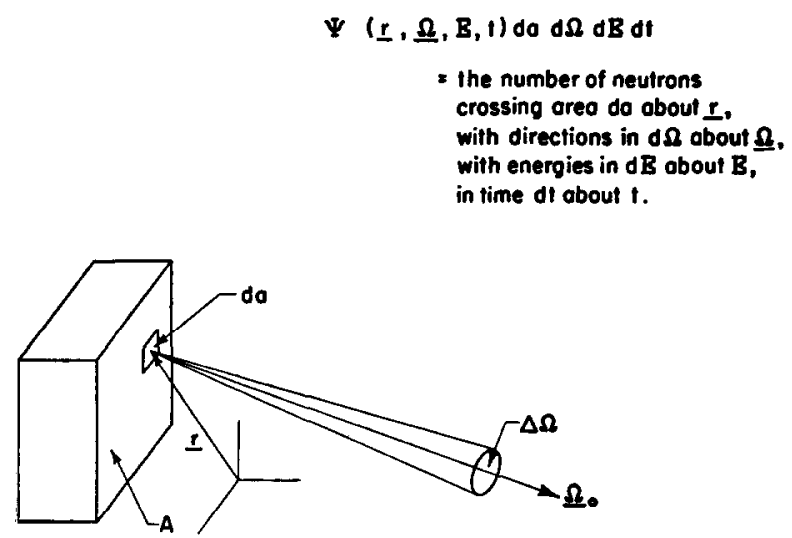

Fig. 1. Definition of the surface angular current density which is measured. enables measurements to be made using a $14 \mathrm{MeV}$ neutron source of $10^{11} \mathrm{n} / \mathrm{sec}$ peak intensity.

Time widths for several polyethylene moderators are reported. The leakage spectra as a function of energy are also measured using conventional time of flight techniques. The absolute yield per incident neutron is measured.

measured, the angular current density at the moderator surface, following injection at high energy

$$
\psi(r, \Omega, E, t) \mathrm{d} a \mathrm{~d} \Omega \mathrm{d} E \mathrm{~d} t
$$

$=$ the number of neutrons crossing area $\mathrm{d} a$ about $r$ with directions in $\mathrm{d} \Omega$ about $\Omega$ with energies in $\mathrm{d} E$ about $E$, in time $\mathrm{d} t$ about $t$.

$\psi(r, \Omega, E, t)$ can be expanded in a set of time eigenfunctions:

$$
\begin{aligned}
\psi(r, \Omega, E, t)=\sum_{i} \psi_{i}(r, \Omega, E) \mathrm{e}^{-\lambda_{i} t} & \\
& +\int_{\lambda_{\min }}^{\infty} \psi(\lambda, r, \Omega, E) \mathrm{e}^{-\lambda t} \mathrm{~d} \lambda,
\end{aligned}
$$

where $\psi_{i}$ are the discrete and $\psi(\lambda, r, \Omega, E)$ the continuum eigenfunctions. The existence and nature of the various modes are discussed by Williams ${ }^{1}$ ) and Corngold ${ }^{2,3}$ ).

In an experiment several modes are excited by the burst of fast neutrons. Thus initially, $\psi$ is not separable in time. However, under certain restrictions, there exists a smallest discrete eigenvalue $\lambda_{0}$, smaller than $\lambda_{\min }$, which dominates at long times after the pulse. The requirement that $\lambda_{0}$ be less than $\lambda_{\min }$ places limits on moderator minimum size. $\lambda_{\min }$ evaluated as $\left(v \sum\right)_{\min }$ is about $3 \times 10^{5} \mathrm{sec}^{-1}$ for polyethylene.

Several experimental techniques have been used to study $\psi$. The time constant of the fundamental mode $\lambda_{0}$, has been measured for a number of moderalors by observing the time dependence of thermal neutrons after the higher modes decay following the injection of a pulse of fast neutrons into the moderator. Beyster's ${ }^{4}$ ) work for light water and Sjöstrand' ${ }^{5}$ ) measurements for polyethylene are examples of this technique. $\lambda_{0}$ as a function of buckling is fitted to the following expansion:

$$
\lambda_{0}=\alpha_{0}+D_{0} B^{2}-C_{0} B^{4}+\ldots
$$

where $\alpha_{0}=v \sum_{\mathrm{a}}$ for $1 / v$ absorption, $D_{0}$ is the diffusion 


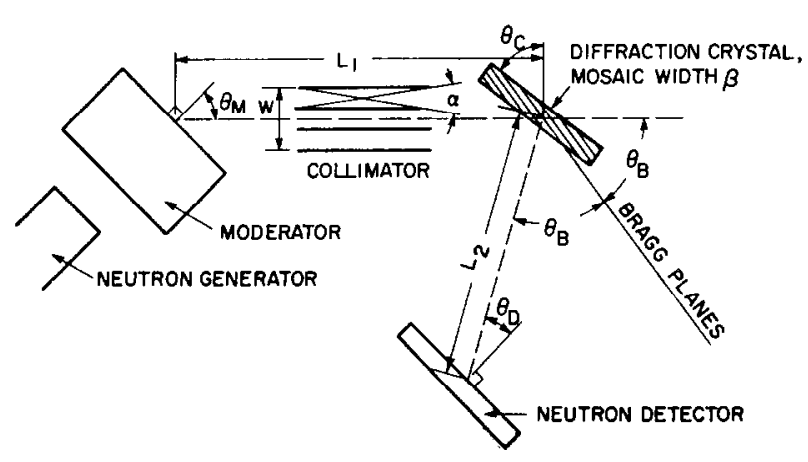

Fig. 2. Schematic view of experimental arrangement.

coefficient and $C_{0}$ is the diffusion cooling constant. The truncated series diverges for small moderators. Sjöstrand studied moderators with bucklings less than $1.4844 \mathrm{~cm}^{-2}$ and observed a maximum value of $\hat{\lambda}_{0}=3.944 \times 10^{4} \mathrm{sec}^{-1}$.

A second technique is the time-of-flight energy spectrum measurement used by Poole ${ }^{6}$ ) and Beyster ${ }^{7}$ ). Here the time integral of $\psi$ is obtained:

$$
\psi(r, \Omega, E)=\int \mathrm{d} t \psi(r, \Omega, E, t) .
$$

Two methods are capable of measuring $\psi(r, \Omega, E, t)$ directly. A neutron chopper phased to open a set time after the fast neutron pulse, coupled with time-of-flight energy measurements has been used by Slovacek ${ }^{8}$ ) and Menzel $^{9}$ ) to measure the neutron energy spectrum at discrete times. Position and neutron direction are set by collimation. This method has required very intense fast neutron sources.

We have used a second method for measuring $\psi(r, \Omega, E, t)$. A crystal spectrometer is arranged to accept neutrons of a fixed energy $E$, in a particular direction $\Omega_{0}$, from a particular position $r$, and the time distribution of the selected neutrons is examined, following a pulse of fast neutrons. This method has also been used by Fluharty ${ }^{10}$ ), Riccobono ${ }^{11}$ ), and Day $^{12}$ ) to study pulsed moderators. Further recent measurements on pulsed moderators have been performed at Brookhaven National Laboratory ${ }^{13}$ ).

\section{Time resolution}

The most severe experimental requirement for the crystal spectrometer technique is adequate time resolution. Neutron speed variations (energy resolution) and flight path length differences cause flight time variations which obscure the time dependence being investigated. Fluharty needed very tight collimation and a crystal with small mosaic width to obtain adequate time resolution. Day was limited by lack of time resolution.
If the spectrometer can be arranged to minimize flight-time variations while sampling larger intervals of $r, \Omega$ and $E$, the measurements can be performed with a more modest neutron source. We present such a "timefocussed" method. The size of the intervals which can be sampled is then determined by the rate of variation of $\psi(r, \Omega, E, t)$ with $r, \Omega$ and $E$.

In our measurements, what is actually determined is the integral of $\psi$, over the moderator surface, averaged over the angular aperture of the detector

$J\left(\Omega_{0}, E, t\right)=\int_{\text {moderator urface }} \mathrm{d} a(1 / \Delta \Omega) \int_{\Delta \Omega} \mathrm{d} \Omega \psi(r, \Omega, E, t)$

which is the total current per unit solid angle about direction $\Omega_{0}$, per unit energy per unit time. $J\left(\Omega_{0}, E, t\right)$ is just what is required to be known for a pulsed moderator to be used as a slow neutron source in connection with a pulsed reactor.

Fig. 2 shows the spectrometer schematically. (Note that in a previous paper ${ }^{14}$ ) the crystal face tilt angle $\theta_{\mathrm{C}}$ was drawn incorrectly.) $\alpha$ is the collimator angular divergence in the scattering plane; $W$ is the collimator lateral beam width at the crystal; and $\beta$ is the angular width of the monochromating crystal reflectivity distribution. The remaining symbols defined on the figure are self-explanatory. The moderator, crystal, and detector planes are normal to the plane of the diagram, which is the scattering plane. The counting rate $C(t)$ is proportional to:

$$
C(t) \sim \psi(r, \Omega, E, t) \cdot \alpha \cdot \beta \cdot W,
$$

so that the largest values of $\alpha, \beta$, and $W$ are clearly desired to produce a high counting rate. The range of energies, angles, and positions over which the spectrometer samples $\psi$ clearly also increases with $\alpha, \beta$, and $W$.

The time variation of $\psi$ in many cases is very rapid and good time resolution is required. The variance $\sigma_{\text {spectrometer }}^{2}$ of the time distribution observed at the detector, for a delta function time distribution at the moderator, is a measure of the time resolution of the spectrometer. A straightforward calculation using the technique of Zweifel and Carpenter ${ }^{15}$ ) gives:

$$
\begin{aligned}
\sigma_{\text {spectrometer }}^{2} & =A \alpha^{2}+B \beta^{2}+C W^{2} \\
& +\sigma_{\text {crystal }}^{2}+\sigma_{\text {detector }}^{2}+\text { higher order terms. }
\end{aligned}
$$

$A, B$ and $C$ are coefficients that depend on system geometry and are given by the following equations:

$$
A=\frac{1}{v_{n}^{2}}\left[\frac{L_{1}+L_{2}}{\tan \theta_{\mathrm{B}}}-L_{1} \tan \theta_{\mathrm{M}}-L_{2} \tan \theta_{\mathrm{D}}\right]^{2},
$$




$$
\begin{aligned}
& B=\frac{1}{v_{n}^{2} \tan ^{2} \theta_{\mathrm{B}}}\left[L_{1}+L_{2}-2 L_{2} \tan \theta_{\mathrm{B}} \tan \theta_{\mathrm{D}}\right]^{2}, \\
& C=\frac{1}{3 v_{n}^{2}}\left[\tan \theta_{\mathrm{M}}-\tan \theta_{\mathrm{C}}+\frac{\sin \left(2 \theta_{\mathrm{B}}+\theta_{\mathrm{C}}+\theta_{\mathrm{D}}\right)}{\cos \theta_{\mathrm{C}} \cos \theta_{\mathrm{D}}}\right]^{2} .
\end{aligned}
$$

Here, $v_{n}$ is the neutron speed for crystal reflection of order $n$. Note that order of reflection appears in no other way.

\section{Time focussing}

Contributions to the time resolution due to collimator angular divergence, crystal mosaic spread, and lateral beam width can be focussed out (made equal to zero) when $A=0, B=0$, and $C=0$. The system geometry must then satisfy three conditions for time focussing: (as written, these do not correspond to conditions $A=0, B=0, C=0$, respectively)

$$
\begin{aligned}
& \tan \theta_{\mathrm{M}}=\frac{1}{2}\left(1+L_{2} / L_{1}\right) \cot \theta_{\mathrm{B}}, \\
& \tan \theta_{\mathrm{D}}=\frac{1}{2}\left(1+L_{1} / L_{2}\right) \cot \theta_{\mathrm{B}}, \\
& \tan \theta_{\mathrm{C}}=\frac{\cos \theta_{\mathrm{D}} \tan \theta_{\mathrm{M}}+\sin \left(2 \theta_{\mathrm{B}}+\theta_{\mathrm{D}}\right)}{2 \sin \theta_{\mathrm{B}} \sin \left(\theta_{\mathrm{B}}+\theta_{\mathrm{D}}\right)} .
\end{aligned}
$$

With these conditions, $\alpha, \beta$, and $W$ can be fairly large without affecting the time resolution. Clearly, since eqs. (6) involve the order of reflection only in a constant of proportionality on $A, B$ and $C$, the conditions $A=B=C=0$ imply focussing of all orders. Similar expressions have been obtained by $\mathrm{Holas}^{16}$ ) for time-of-flight diffractometry.

The remaining terms in eq. (5) must be considered. $\sigma_{\text {crystal }}^{2}$ and $\sigma_{\text {detcctor }}^{2}$ are due to the physical thickness of the crystal and detector. Each must be thin enough that their neutron transit time dispersion does not contribute significantly to the time resolution. The pulse width, $\sigma_{\text {source }}$, of the fast neutron source must also be small, since the instrumental contribution to the observed time variance is $\sigma_{\text {instr. }}^{2}=\sigma_{\text {spect. }}^{2}+\sigma_{\text {source }}^{2}$

Higher order terms of eq. (5) may be significant when the first order terms are focussed out. We have found that these terms are at least an order of magnitude smaller than the unfocussed first order terms, for our measurements described below.

Flight time variations due to components of the neutron direction orthogonal to the scattering plane are time-focussed providing the moderator face, crystal face and detector plane are all orthogonal to the scattering plane. This eliminates effects due to the collimator height, and vertical angular divergence.

The three equations for time focussing allow the experimentalist some freedom as there are at least five variable parameters - thus allowing certain other experimental constraints to be satisfied. We used a crystal whose Bragg planes were parallel to the plane of the crystal. The focussing conditions then specify that the moderator face, crystal face, crystal Bragg and detector face planes be parallel and $L_{1}=L_{2}$. It is unfortunately impossible to make the moderator tilt angle zero and satisfy all focussing conditions, but it is possible to reduce this angle by making $L_{1} \gg L_{2}$ and using a large Bragg angle. This, however, necessitates a more acute detector tilt angle and the use of a crystal cut at an angle to the reflecting planes.

\section{Partial focussing}

In some cases it may not be necessary to use complete focussing. For example the contribution to time resolution from crystal mosaic width may be small compared to the other terms. If $A$ and $C$ in eq. (5) are set equal to zero the following partial focussing equations result:

$$
\begin{aligned}
& \tan \theta_{\mathrm{M}}=\tan \theta_{\mathrm{C}}-\frac{\sin \left(2 \theta_{\mathrm{B}}+\theta_{\mathrm{C}}+\theta_{\mathrm{D}}\right)}{\cos \theta_{\mathrm{C}} \cos \theta_{\mathrm{D}}}, \\
& \frac{L_{1}+L_{2}}{\tan \theta_{\mathrm{B}}}=L_{1} \tan \theta_{\mathrm{M}}+L_{2} \tan \theta_{\mathrm{D} .}
\end{aligned}
$$

These allow one additional degree of freedom. One then can observe the moderator normal to its surface and satisfy these conditions. Or the condition $L_{1}=L_{2}$, which arises in the fully focussed case when the crystal Bragg planes are parallel to the crystal face, can be relaxed.

\section{Other considerations}

The total length of the neutron flight path from moderator to detector must satisfy certain constraints. The neutron time distribution observed at the detector is delayed from that leaving the moderator by the time of flight. This delay time is inversely proportional to the order of crystal reflection thus separating the observed time distributions for each corresponding energy. The total flight path length must be sufficiently long to prevent overlap of the various orders.

Long flight paths have an important detrimental effect on the rate of data collection. Due to the low yield of our accelerator, we were forced to use the minimum possible flight path to obtain sufficient data collection rates. Time resolution is also adversely affected by long flight paths. Without focussing, the time resolution width $\sigma_{\text {spectrometer }}$ is roughly proportional to total path length. With time focussing the dominant terms have been nulled out, but contributions due to imperfect 


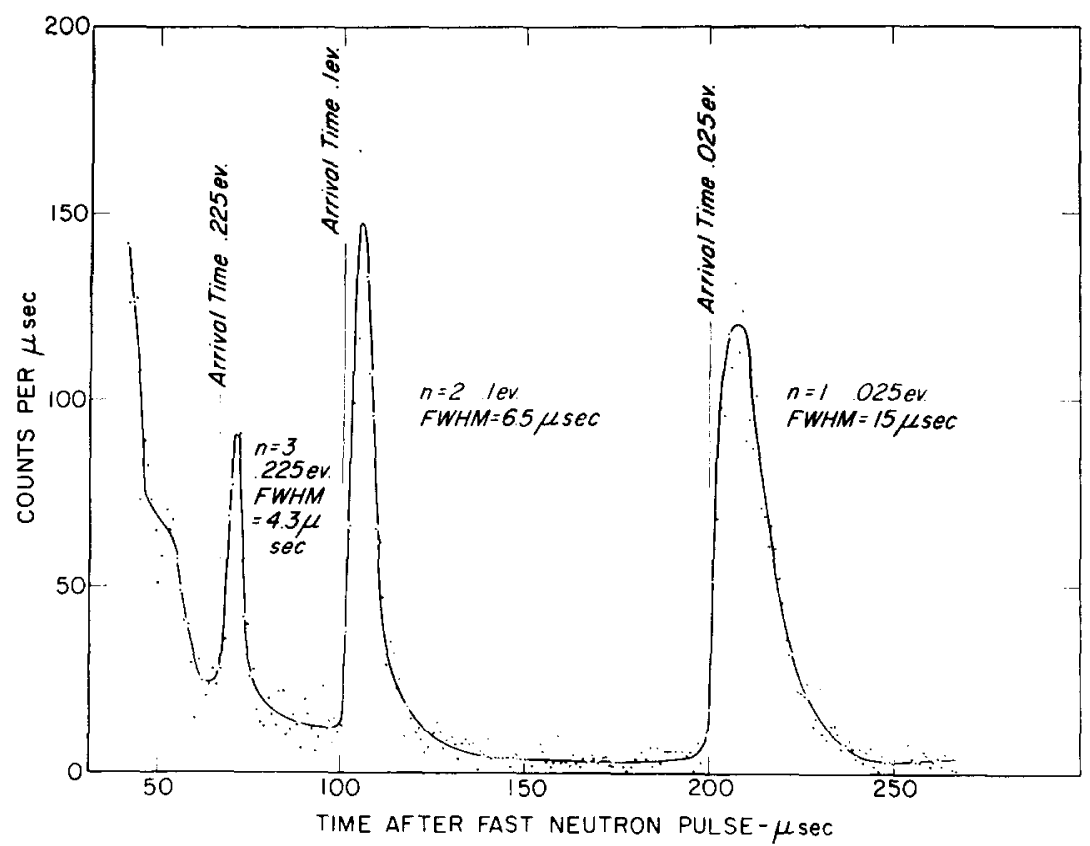

Fig. 3. Raw data for the time-focussed condition for $7.65 \mathrm{~cm} \times 10 \mathrm{~cm} \times 10 \mathrm{~cm}$ polyethylene, heterogeneously poisoned with $0.05 \mathrm{~cm}$ thick cadmium, $1.0 \mathrm{~cm}$ beneath the viewed surface. Temperature $=23^{\circ} \mathrm{C} . L_{1}=L_{2}=21.95 \mathrm{~cm} ; \theta_{\mathrm{B}}=30^{\circ} ; \theta_{\mathrm{M}}=\theta_{\mathrm{C}}=\theta_{\mathrm{D}}=60^{\circ}$.

focussing and the higher order terms are still proportional to the total path length.

\section{Description of the time-focussed crystal spectrometer}

We have performed measurements using a Texas Nuclear Neutron Generator producing D-T neutrons $\left(14.7 \mathrm{MeV}\right.$ at $10^{11} \mathrm{n} / \mathrm{sec}$ peak). In these experiments the counting rates were still rather low, even using the increased spectrometer efficiency made possible by time-focussing. Therefore, it was ncecssary to reduce background to a low level. Several steps were taken to accomplish this. The neutron generator, normally equipped with both pre- and post-acceleration deflection pulsing, was equipped with a device ${ }^{17}$ ) to turn off ion beam extraction voltage between pulses. This reduced the beam turn-off from about $10^{-5}$ to a level smaller than $10^{-7}$.

Further, to maintain low background, the crystal and detector were housed within a cadmium-lined box with $3^{\prime \prime}$ thick walls of $\mathrm{B}_{4} \mathrm{C}, 4^{\prime \prime} \times 11^{\prime \prime} \times 17^{\prime \prime}$ inside dimensions.

The detector used was a $3^{\prime \prime}$ diameter $\times 0.027^{\prime \prime}$ uncorrugated flat plate ${ }^{6} \mathrm{LiF}-\mathrm{ZnS}$ "Stedman" type neutron scintillation counter ${ }^{18}$ ) on a $\frac{1}{2}$ " pyrex light guide attached to a RCA 4524 photomultiplier, with pulse shape discrimination ${ }^{19}$ ) for gamma ray pulse rejection. We found a high-efficiency lithium glass scintillator unsuitable because of its high natural background, high gamma ray sensitivity, and primarily because of silicon activation from $14 \mathrm{MeV}$ neutrons.

With these arrangements, the background with the accelerator not operating is 0.15 counts $/ \mathrm{min}$; it is 0.3 counts/min between pulses, with no moderator, when the accelerator is operating.

The crystal is copper of dimensions $1 \frac{1}{2}^{\prime \prime} \times 4 \frac{1}{2}^{\prime \prime} \times 0.125^{\prime \prime}$, cut parallel to the $(200)$ planes $(d=1.804 \AA)$, whose rocking curve width is about 30 minutes of $\operatorname{arc}(\mathrm{fwhm})$. A multi-slit Soller collimator of dimensions $W=2^{\prime \prime}$, $H=1.65^{\prime \prime}$, length $-4.6^{\prime \prime}$ and a slit width of $0.28^{\prime \prime}$ gives an angular beam divergence of $3.5^{\circ}(\mathrm{fwhm})$ and samples a large fraction of the surface of a tilted $10 \mathrm{~cm} \times 10 \mathrm{~cm}$ moderator face. The energy resolution is typically $\approx 20 \%$ being dominated by the collimation.

Fig. 3 shows a typical time distribution measured with the fully-focussed apparatus. The moderator is a $7.65 \mathrm{~cm} \times 10 \mathrm{~cm} \times 10 \mathrm{~cm}$ polyethylene block with a $0.05 \mathrm{~cm}$ cadmium sheet $1.0 \mathrm{~cm}$ beneath the viewed surface. Three peaks are easily distinguished, each due to reflection in a different order from the crystal,

$$
\lambda_{n}=2 d \sin \theta_{\mathbf{B}} / n \text {. }
$$

Here $\lambda=h / m v$ is the neutron wavelength, $d$ is the plane spacing, $\theta_{\mathrm{B}}$ is the Bragg angle, and $n$ the order of reflection. With $\theta_{\mathrm{B}}=30^{\circ}$, the nominal neutron energies are $0.025,0.1$, and $0.225 \mathrm{eV}$ for $n=1,2,3$. The three peaks are displaced from the time of emission from the moderator, by their flight time over the $43.9 \mathrm{~cm}$ total 


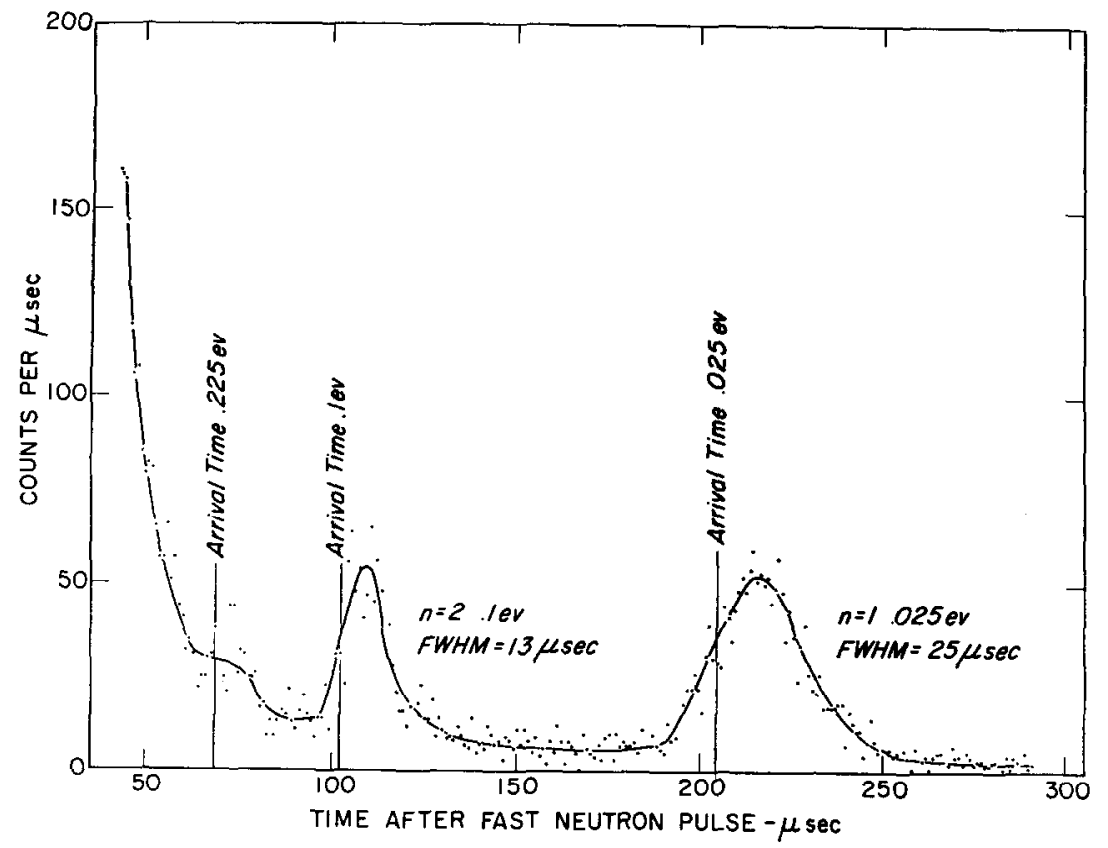

Fig. 4. Raw data for the unfocussed condition for the same moderator as in fig. $3 . L_{1}=L_{2}=22.35 \mathrm{~cm} ; \theta_{13}=30^{\circ} ; \theta_{\mathrm{M}}=0_{\mathrm{D}}=0^{\wedge} ; O_{\mathrm{C}}=60^{\circ}$

flight path. The moderator is tilted such that $\theta_{M}=60^{\circ}$; thus $J\left(\Omega_{0}, E, t\right)$ is measured with $\Omega_{0}$ being $60^{\circ}$ from normal to the viewed face. The detector angle is also $60^{\circ}$.

The observed full width at half maximum, fwhm, are $4.3 \mu \mathrm{sec} \pm 0.5 \mu \mathrm{sec}$ for the $0.225 \mathrm{eV}$ peak, $6.5 \mu \mathrm{sec} \pm 0.5$ $\mu \mathrm{sec}$ for the $0.1 \mathrm{eV}$ peak, and $15 \mu \mathrm{sec} \pm 1 \mu \mathrm{sec}$ for the $0.025 \mathrm{eV}$ peak. Broadening is primarily due to the $2.5 \mu \mathrm{sec}$ long $14.7 \mathrm{MeV}$ neutron source pulse. The time scale is measured from the center of this source pulse.
Calculated flight times for each peak are shown and are based on the nominal energies and centerline distances. The start of each peak is within $1 \mu \mathrm{sec}$ of the calculatcd value and the initial rise is very rapid.

\section{Unfocussed spectrometer}

Fig. 4 shows the time distribution from the same moderator, but measured with the apparatus in an unfocussed geometry. $\theta_{\mathrm{M}}$ and $\theta_{\mathrm{D}}$ were zero - i.e. both

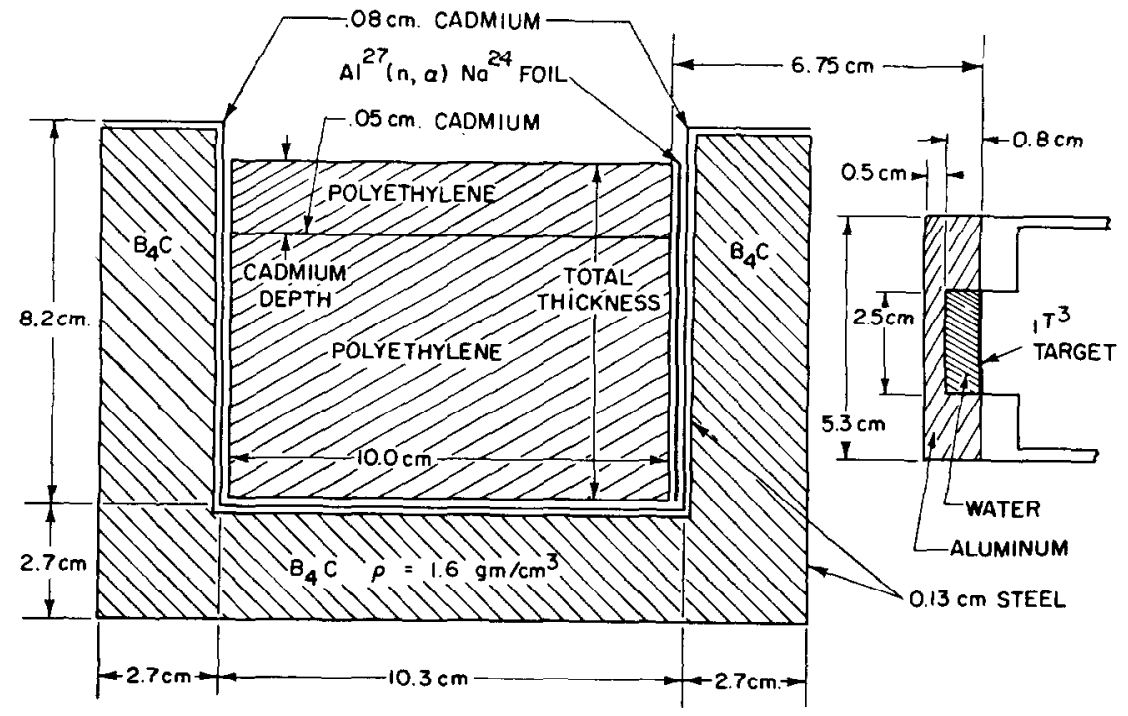

Fig. 5. Detailed arrangement of moderator, neutron source and shielding. 

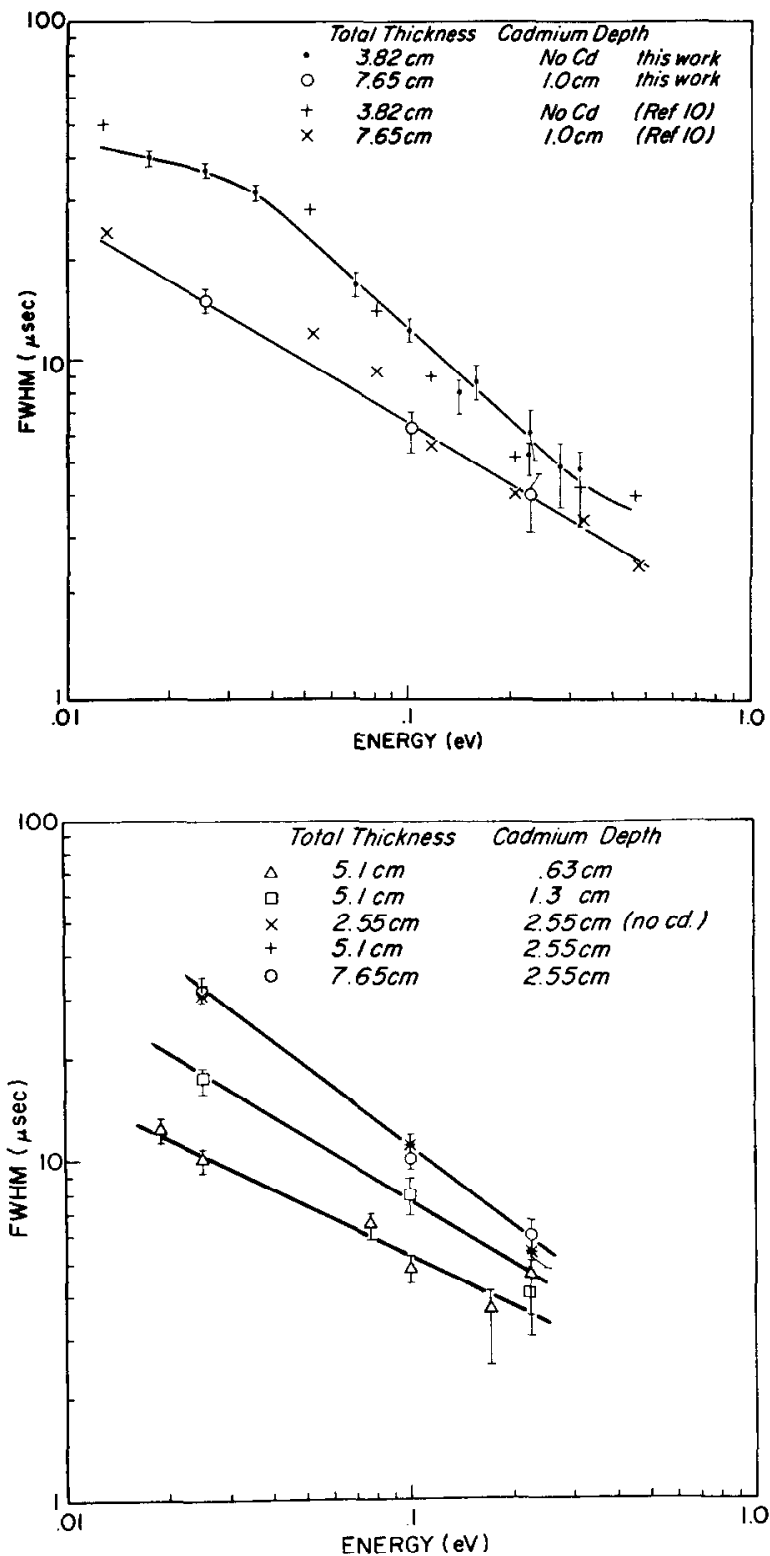

Fig. 6a, b. Measured full widths at half maximum (fwhm) vs neutron energy for various $23^{\circ} \mathrm{C}$ polyethylene moderators of $10 \mathrm{~cm} \times 10 \mathrm{~cm}$ lateral dimensions.

the detector and moderator faces were normal to the nominal neutron direction. The total flight path length was increased slightly to $44.7 \mathrm{~cm}$. Except for these changes, the geometry was identical with that for the focussed condition. It is clear that there is considerable degradation of the time resolution in this unfocussed geometry. The fwhm are broadened to $13 \mu \mathrm{sec} \pm 1 \mu \mathrm{sec}$ for the $0.1 \mathrm{eV}$ peak and $25 \mu \mathrm{sec} \pm 2 \mu \mathrm{sec}$ for the $0.025 \mathrm{eV}$ peak. The $0.225 \mathrm{eV}$ peak is poorly resolved.

The $0.025 \mathrm{eV}$ time distribution starts about $10 \mu \mathrm{sec}$ before the calculated time of flight and has a much slower initial rise than for the focussed case. The $0.1 \mathrm{eV}$ peak shows similar distortion.

Within the quoted error limits, for both the 0.025 and $0.1 \mathrm{eV}$ peaks,

$$
\sigma_{\text {unfocussed }}^{2}=\sigma_{\text {focussed }}^{2}+(\Delta t)^{2},
$$

where $(\Delta t)^{2}$ is the change in time resolution between focussed and unfocussed conditions calculated using eq. (5).

\section{Measurements of time distributions of polyethylene moderators}

This time focussed apparatus is being used to study various moderators. Our main purpose here is to describe the time focussed crystal spectrometer, so only some representative results will be given, without detailed interpretation.

Fig. 5 shows the moderator arrangement for a series of measurements on $10 \mathrm{~cm} \times 10 \mathrm{~cm}$ square blocks of room-temperature polyethylene (density $0.92 \mathrm{~g} / \mathrm{cm}^{3}$ ). The $\mathrm{B}_{4} \mathrm{C}$-filled, Cd-lined box surrounds the moderator block on all sides except the viewed face. Measurements were performed on a series of moderators of $2.55 \mathrm{~cm}$ to $7.65 \mathrm{~cm}$ total thickness. Some moderators were heterogeneously poisoned with $10 \mathrm{~cm} \times 10 \mathrm{~cm} \times 0.05 \mathrm{~cm} \mathrm{Cd}$ placed at various depths beneath the viewed surface. Fig. 6 summarizes the results in terms of the measured full width at half maximum, fwhm, plotted vs energy for seven moderators. Fluharty's ${ }^{10}$ ) results for two of the same moderator geometries are shown for com-

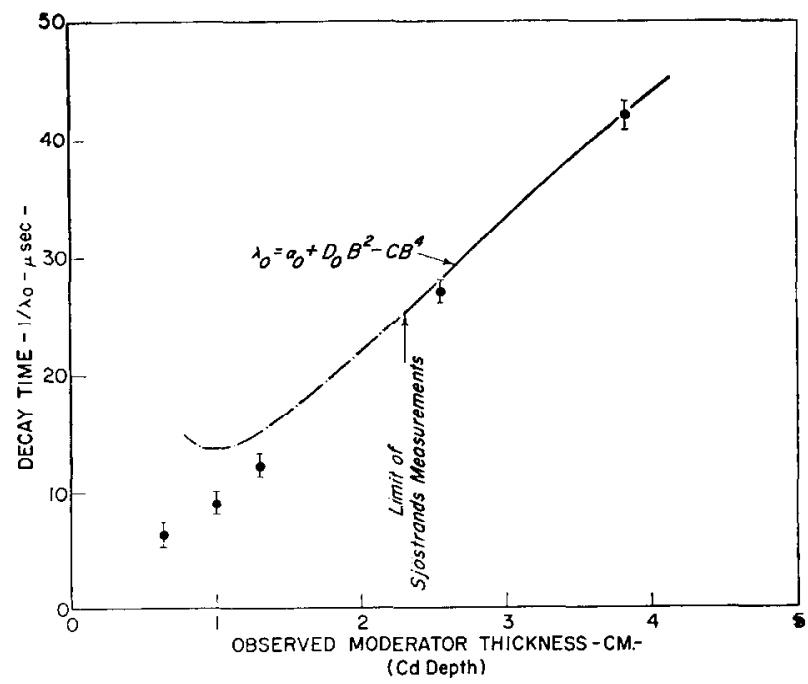

Fig. 7. Observed persistant decay times vs cadmium depth for $23^{\circ} \mathrm{C}$ polyethylene moderators of $10 \mathrm{~cm} \times 10 \mathrm{~cm}$ lateral dimensions. 
parison. Below $0.1 \mathrm{eV}$, these are generally somewhat larger than a curve through our values. Our measurements were made for neutrons emerging at a considerable angle from normal to the moderator face. Fluharty's measurements were made normal to the face. Also different spatial modes of the slow neutron distribution may have been excited due to the larger mean free path of the $14.7 \mathrm{MeV}$ neutrons than of the roughly $2 \mathrm{MeV}$ neutrons from the linac ${ }^{9}$ ). These experimental differences may explain the noted differences in pulse widths.

The only correction to the data is for the width of the fast neutron pulse and analysis channel width. Except for the fwhm below $10 \mu \mathrm{sec}$, this correction is negligible. The following equation is used:

$$
\begin{aligned}
& \mathrm{fwhm}_{\text {corrected }}^{2}=\mathrm{fwhm}_{\text {observed }}^{2} \\
& \quad-\left[(1.96)^{2} / 12\right][\text { pulse width } \\
&
\end{aligned}
$$

The factor $(1.96)^{2} / 12$ is obtained by treating the observed and corrected time distributions as having the shape of the infinite medium, free-proton slowing down time distribution ${ }^{20}$ ), which has a functional dependence of $x^{2} \mathrm{e}^{-x}$. The fast neutron pulse and channel widths are rectangular. Error limits shown are estimated uncertainties in this correction and in extracting the observed fwhm.

Fig. 7 shows the decay time of the exponential "tail" of the pulse following the initial transient. This is plotted vs viewed polyethylene thickness above the $\mathrm{Cd}$. With certain reservations, this may be considered as the fundamental decay time $1 / \lambda_{0}$. The tails were only observable for 1 to 2 decades with our low intensity neutron source so no claim can be made that the decay represents only a single exponential. This is especially true for the thinner moderators. No corrections have been applied to these observed decay times.

For the thickest unpoisoned moderator studied $(3.82 \mathrm{~cm})$, the same decay time was observed for the time distributions at $0.018,0.025,0.035,0.076$, and $0.1 \mathrm{eV}$. At higher energies the magnitude of the tail was too small to reasonably measure its period. For thinner moderators, the tail decay times werc only measurable for the first order peak (generally $0.025 \mathrm{eV}$ ).

The decay time obtained agrees with that obtained by Day $^{12}$ ) for the $3.82 \mathrm{~cm}$ thick moderator when correction is made for different transverse buckling. For the thinner moderators, our values for $1 / \lambda_{0}$ are significantly smaller and reach a factor of 2 smaller for $0.63 \mathrm{~cm}$ thick moderator. We attribute this to superior time resolution in the present measurements.

Our results also comparc with those predicted by

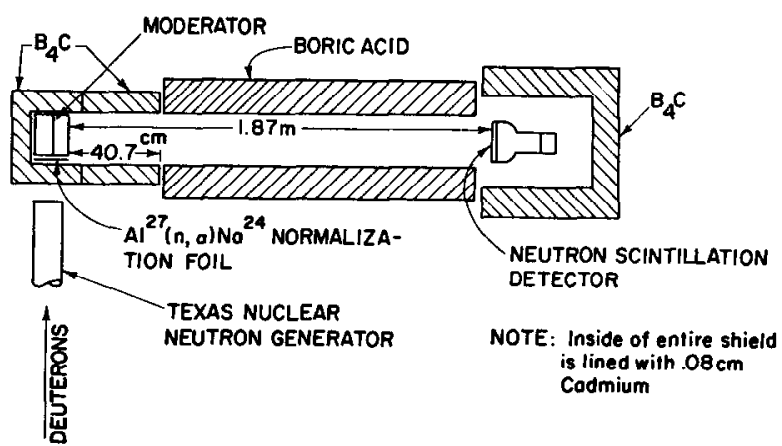

Fig. 8. Experimental arrangement of time-of-flight spectrometer.

using Sjöstrand's ${ }^{5}$ ) coefficients in the buckling power series expansion for $\lambda_{0}$ [eq. (2)]. Again the agreement is good for the thicker two moderators. For thinner moderators, with large buckling, the truncated series expansion clearly diverges. As mentioned previously, a discrete $1 / \lambda_{0}$ may exist down to a value of about $3 \mu \mathrm{sec}$ for polyethylene. The time distributions at the energies measured (all below the cadmium cutoff) were found to be unaffected by the presence or thickness of moderator behind the cadmium, depending only on the thickness of moderator above the $\mathrm{Cd}$.

\section{Absolute energy spectra}

A second, parallel, series of experiments was performed to measure absolutely the leakage spectra as a function of energy by conventional time-of-flight techniques. This is related to $\psi$ by

$J\left(\Omega_{0}, E\right)=\int_{\text {moderator face }} \mathrm{d} a \frac{1}{\Delta \Omega} \int_{\Lambda \Omega} \mathrm{d} \Omega \int \mathrm{d} t \psi(r, \Omega, E, t)$,

where $t$ is the neutron emission time following the fast neutron burst. The experiment was performed since the diffraction experiment does not provide absolute yields.

\section{Description of the time-of-flight spectrometer}

Fig. 8 shows the overall experimental arrangement while fig. 5 shows a detailed view of the moderator, moderator shield box, and neutron generator target. The moderator is viewed normal to its surface while the incident $14.7 \mathrm{MeV}$ neutrons enter the side of the moderator. The $14.7 \mathrm{MeV}$ neutron source is effectively a disc source of $1 \mathrm{~cm}$ diameter located $6.75 \mathrm{~cm}$ from and centered on the side of the polyethylene moderator. This source is nearly isotropic over the range of angles involved.

An aluminum activation foil the same dimensions as the side of the moderator is used to measure the in- 


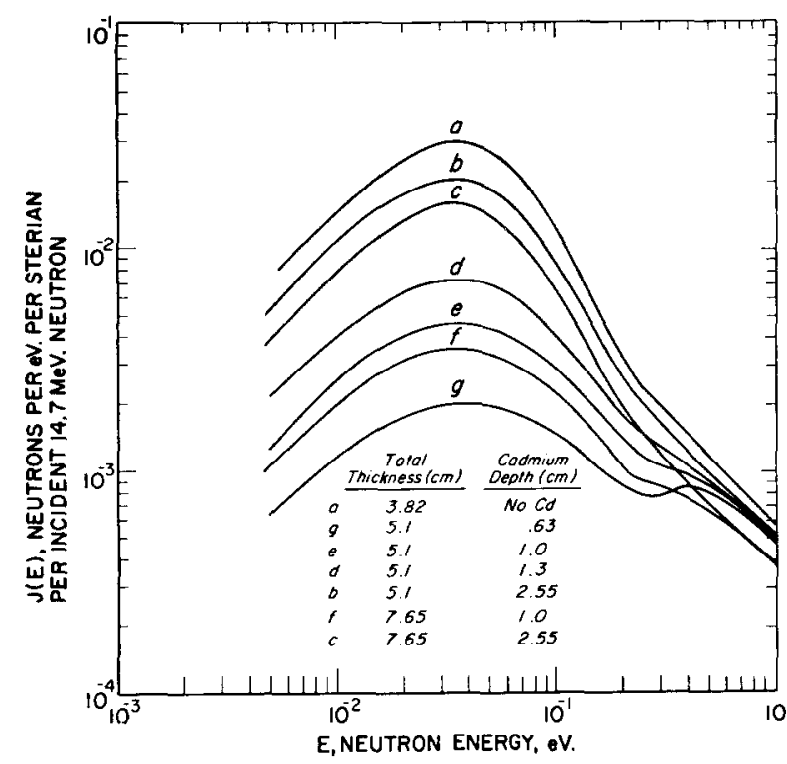

Fig. 9. Energy spectra for various $23^{\circ} \mathrm{C}$ polyethylene moderators of $10 \mathrm{~cm} \times 10 \mathrm{~cm}$ lateral dimensions.

cident number of $14.7 \mathrm{MeV}$ neutrons. The foils are activated by the ${ }^{27} \mathrm{Al}(\mathrm{n}, \alpha)^{24} \mathrm{Na}$ reaction with a cross section of $115 \mathrm{mb}$ at $14.7 \mathrm{MeV}$ and an effective threshold energy of $6 \mathrm{MeV}$. Neutrons backscattering from polyethylene are very nearly all below the threshold. The $15 \mathrm{~h}, 2.75 \mathrm{MeV}^{24} \mathrm{Na}$ gamma activity is determined using a $3^{\prime \prime} \times 3^{\prime \prime} \mathrm{NaI}$ crystal and efficiencies from Heath ${ }^{21}$ ). The average incident neutron fluence determined from the foil measurement is multiplied by the area of the side of the moderator to obtain the incident number of $14.7 \mathrm{MeV}$ neutrons. No correction is made for epi-threshold scattered neutrons causing activation in the foil; the activation is treated as though all activations are caused by $14.7 \mathrm{MeV}$ neutrons. The geometry in fig. 5 is given to allow corrections to be calculated.

Early measurements were performed without the $\mathrm{B}_{4} \mathrm{C}$ shielding around the moderator. The moderator was shielded on 5 sides with $0.08 \mathrm{~cm}$ cadmium and there was a gap of about $40 \mathrm{~cm}$ between the moderator and the Boric acid crystal filled shield. Measurements of the transmission of an indium foil showed serious and increasing discrepancies below $0.025 \mathrm{eV}$. This was attributed to room return neutrons scattering from the moderator and arriving at the detector with energies higher than prescribed by their arrival time.

With the $\mathrm{B}_{4} \mathrm{C}$ shielding the measurement of the indium cross section agreed with published values to below $0.01 \mathrm{eV}$ where statistics became poor. The measured absolute yield of the $\mathrm{B}_{4} \mathrm{C}$ shielded moderators increased by a factor of 2.1 over the $\mathrm{Cd}$ shielded moderator when compared above $0.025 \mathrm{eV}$. This is a measure of the reflection of high energy neutrons into the moderator by the $\mathrm{B}_{\mathbf{4}} \mathrm{C}$ shielding.

The detector used for the spectrum measurements is the same scintillation detector previously described. The efficiency of this detector was obtained by comparing it with a ${ }^{6} \mathrm{Li}$ glass scintillator (Nuclear Enterprises NE 912) whose transmission we measured using the University of Michigan chopper at the Ford Nuclear Reactor. Above $0.01 \mathrm{eV}$ the efficiency of the detector was determined to within $10 \%$, but below $0.01 \mathrm{eV}$ the high background of the glass scintillator prevented an accurate determination.

\section{Measurements of absolute energy spectra of polyethylene}

The results of the energy spectrum measurements are

TABle 1

Yields and "effective temperature" for polyethylene moderators $\left(\rho=0.92 \mathrm{~g} / \mathrm{cm}^{3}\right)$.

\begin{tabular}{|c|c|c|c|c|}
\hline Moderator size $(\mathrm{cm})$ & $\begin{array}{l}\text { Cadmium depth } \\
(\mathrm{cm})\end{array}$ & $\begin{array}{c}\text { "Effective } \\
\text { temperature" } \\
E_{\mathrm{Th}}(\mathrm{eV})\end{array}$ & Yield $\boldsymbol{J}_{\mathrm{Th}}$ & $\int_{0.01 \mathrm{eV}}^{0.1} \mathrm{ev} J(E) \mathrm{d} E$ \\
\hline $3.82 \times 10 \times 10$ & No $\mathrm{Cd}$ & 0.0325 & $2.44 \times 10^{-3}$ & $1.86 \times 10^{-3}$ \\
\hline $5.1 \times 10 \times 10$ & 0.63 & {$\left[\begin{array}{l}0.042 \\
0.036\end{array}\right.$} & $\left.\begin{array}{l}0.215 \times 10^{-3} \\
0.182 \times 10^{-3}\end{array}\right]$ & $0.147 \times 10^{-3}$ \\
\hline $5.1 \times 10 \times 10$ & 1.0 & 0.037 & $0.43 \times 10^{-3}$ & $0.32 \times 10^{-3}$ \\
\hline $5.1 \times 10 \times 10$ & 1.3 & 0.036 & $0.67 \times 10^{-3}$ & $0.50 \times 10^{-3}$ \\
\hline $5.1 \times 10 \times 10$ & 2.55 & 0.033 & $1.67 \times 10^{-3}$ & $1.26 \times 10^{-3}$ \\
\hline $7.65 \times 10 \times 10$ & 1.0 & 0.037 & $0.34 \times 10^{-3}$ & $0.25 \times 10^{-3}$ \\
\hline $7.65 \times 10 \times 10$ & 2.55 & 0.034 & $1.32 \times 10^{-3}$ & $0.98 \times 10^{-3}$ \\
\hline
\end{tabular}

The units of both $J_{\mathrm{Th}}$ and $\int_{0.01 \mathrm{ev}}^{0.1 \mathrm{eV}} J(E) \mathrm{d} E$ are slow neutrons per steradian per incident 14.7 MeV neutron. 
given in fig. 9 and table 1 . These measurements were made normal to the moderator face. Fig. 9 shows the total number of neutrons leaving the moderator face per steradian per electron volt per $14.7 \mathrm{MeV}$ neutron entering the moderator through the foil, as a function of neutron energy for various moderators.

Below the cadmium cutoff, moderators of equal overall dimensions have increasing yields as the depth of the cadmium sheet beneath the surface increases. Above the cadmium cutoff, moderators of identical overall dimensions approach the same yields. The shapes of the spectra below the cadmium cutoff were found to be strongly dependent on the depth of the cadmium sheet and independent of the overall thickness.

The energy spectra were compared to a Maxwellian by graphically fitting an exponential to $J(E) / E$. For all except the $0.63 \mathrm{~cm}$ thick moderator, the spectra have reasonably well-defined Maxwellian components. In the $0.63 \mathrm{~cm}$ case, no single Maxwellian acceptably fits the spectrum. Below $0.04 \mathrm{eV}$ a $0.036 \mathrm{eV}$ Maxwellian fits the data while above $0.04 \mathrm{eV}$, the effective temperature is $0.042 \mathrm{eV}$. In all cases, the "effective temperature" of the leakage flux is significantly above $k T=0.0254 \mathrm{eV}$, which was the physical moderator temperature, and the effective temperature increases with decreasing cadmium depth.

Two energy-integrated yields are given in table 1 . The units of both are neutrons per steradian per incident 14.7 MeV neutron entering the moderator. One is the experimentally measured leakage current integrated between 0.01 and $0.1 \mathrm{eV}$. The other is the total in the Maxwellian component, obtained from the expression

$$
J_{\mathrm{Th}}=E_{\mathrm{Th}}^{2} \lim _{E \rightarrow 0}[J(E) / E] .
$$

Here, $E_{\mathrm{Th}}$ is the determined Maxwellian temperature.

These absolute yields must be used and interpreted very carefully. The results apply for the geometry and source studied; for $14.7 \mathrm{MeV}$ neutrons incident as shown in fig. 5 on a polyethylene moderator surrounded on 5 sides with $2.5 \mathrm{~cm}$ of $\mathrm{B}_{4} \mathrm{C}$ whose density is about $1.6 \mathrm{~g}$ per $\mathrm{cm}^{3} . \Lambda$ s previously discussed, the presence of the $\mathrm{B}_{4} \mathrm{C}$ shield increases the yield by a factor of 2.1 .

The yields for different total thickness moderators for the same cadmium depth can be misleading. As shown per incident $14.7 \mathrm{MeV}$ neutron, the yield of the $5.1 \mathrm{~cm}$ total thickness moderators is higher by a factor of 1.3 than for the $7.65 \mathrm{~cm}$ thick moderators with the same cadmium depth. However, per $14.7 \mathrm{MeV}$ neutron incident per $\mathrm{cm}^{2}$ on the moderator, the yield of the thicker moderator is higher by a factor of 1.15 .

\section{Summary}

In summary, we have described a high-efficiency, time-focussed crystal spectrometer useful with lowintensity neutron sources to study time distributions at chosen energies. We have also described a method by which absolute slow neutron yields can be determined. Results of a series of measurements on room temperature polyethylene are presented. These demonstrate the usefulness of the method.

Further studies are being performed on low temperature moderators and moderators heterogeneously poisoned with gadolinium to achieve a lower cutoff energy.

\section{References}

1) M. M. R. Williams, The slowing down and thermalization of neutrons, ch. IV (North-Holland Publ. Co., Amsterdam, 1966).

2) N. Corngold, Nucl. Sci. Eng. 19 (1964) 80.

3) N. Corngold and P. Michael, Nucl. Sci. Eng. 19 (1964) 91.

$\left.{ }^{4}\right)$ W. M. Lopez and J. R. Beyster, Nucl. Sci. Eng. 12 (1962) 190.

5) N. G. Sjöstrand, J. Mednis and T. Nilsson, Arkiv Fysik 15 (1959) 471.

$\left.{ }^{6}\right)$ M. J. Poole and E. R. Wiblin, Proc. 2nd Intern. Conf. Peaceful uses of atomic energy 14 (1958) 266, p/59.

7) J. R. Beyster, J. L. Wood, W. M. Lopez and R. B. Walton, Nucl. Sci. Eng. 9 (1961) 168.

${ }^{8)}$ R. E. Slovacek, Measurement of the asymptotic neutron spectrum in a multiplying medium, Ph.D. Thesis (Rensselaer Polytechnic Institute, Troy, N.Y., 1964).

$\left.{ }^{9}\right)$ J. H. Menzel, Measurements of time-dependent neutron spectra in $\mathrm{H}_{2} \mathrm{O}$, Ph.D. Thesis (Rensselaer Polytechnic Institute, Troy, N.Y., 1968).

10) R. G. Fluharty, F. B. Simpson, G. J. Russell and J. H. Menzel, Nucl. Sci. Eng. 35 (1969) 45.

11) G. Riccobono, G. Fraysse and S. Menardi, Energia Nucl. 15 (1968) 717.

12) D. H. Day and R. N. Sinclair, Nucl. Instr. and Meth. 72 (1969) 237.

13) K. C. Hoffman and J. M. Hendrie, BNL-13605 (1969).

14) J. M. Carpenter and K. F. Graham, Trans. Am. Nucl. Soc. $12(1969) 191$.

15) P. F. Zweifel and J. M. Carpenter, IAEA Conf. Inelastic scattering of neutrons in solids and liquids (Vienna, 1961) p. 199.

16) A. Holas, Nukleonika 13 (1968) 871.

17) J. L. Shapiro, Trans. Am. Nucl. Soc. 11 (1968) 584.

18) R. Stedman, CR R P-931 (1960).

$\left.{ }^{19}\right)$ L. A. Wraight, D. H. C. Harris and P. A. Egelstaff, Nucl. Instr. and Meth. 33 (1965) 181.

$\left.{ }^{20}\right)$ K. H. Beckurts and K. Wirtz, Neutron physics (SpringerVerlag, New York, 1964) p. 168.

21) R. L. Heath, IDO-16880 (1964). 